\title{
Carrier-density-dependent recombination dynamics of excitons and electron-hole plasma in $m$-plane InGaN/GaN quantum wells
}

\author{
W. Liu, ${ }^{1, *}$ R. Butté, ${ }^{1}$ A. Dussaigne, ${ }^{2}$ N. Grandjean, ${ }^{1}$ B. Deveaud, ${ }^{1}$ and G. Jacopin ${ }^{1, \dagger}$ \\ ${ }^{1}$ Institute of Physics, École Polytechnique Fédérale de Lausanne (EPFL), CH-1015 Lausanne, Switzerland \\ ${ }^{2}$ University of Grenoble Alpes, CEA, LETI, MINATEC campus, F-38054 Grenoble, France \\ (Received 8 July 2016; revised manuscript received 4 October 2016; published 10 November 2016)
}

\begin{abstract}
We study the carrier-density-dependent recombination dynamics in $m$-plane InGaN/GaN multiple quantum wells in the presence of $n$-type background doping by time-resolved photoluminescence. Based on Fermi's golden rule and Saha's equation, we decompose the radiative recombination channel into an excitonic and an electron-hole pair contribution, and extract the injected carrier-density-dependent bimolecular recombination coefficients. Contrary to the standard electron-hole picture, our results confirm the strong influence of excitons even at room temperature. Indeed, at $300 \mathrm{~K}$, excitons represent up to $63 \pm 6 \%$ of the photoexcited carriers. In addition, following the Shockley-Read-Hall model, we extract the electron and hole capture rates by deep levels and demonstrate that the increase in the effective lifetime with injected carrier density is due to asymmetric capture rates in presence of an $n$-type background doping. Thanks to the proper determination of the density-dependent recombination coefficients up to high injection densities, our method provides a way to evaluate the importance of Auger recombination.
\end{abstract}

DOI: 10.1103/PhysRevB.94.195411

\section{INTRODUCTION}

$c$-plane InGaN/GaN quantum wells (QWs) are the prevailing active layers in III-nitride-based light-emitting diodes (LEDs), which already achieve high luminous efficiency for white LEDs $(\sim 100 \mathrm{~lm} / \mathrm{W})$ and long lifetime $(\sim 10000 \mathrm{~h})$ $[1,2]$. However, because of the large built-in polarization field $(\sim \mathrm{MV} / \mathrm{cm})$ along the growth direction, the well thickness of $c$-plane QWs is typically limited to $2-3 \mathrm{~nm}$ to maintain a sufficient radiative recombination rate [3]. Note that at high current injection, such a narrow well thickness results in a high carrier density, which enhances the efficiency droop [4]. Thanks to the absence of any built-in polarization field, wider well thicknesses can be used with nonpolar $m$-plane InGaN/GaN QWs, which therefore possess a great potential for LED applications [5], in particular to reduce the influence of the efficiency droop [6]. In addition, because of their in-plane asymmetry, $m$-plane InGaN/GaN QWs are strong candidates for the realization of polarized light sources with an improved efficiency and promising properties for liquid-crystal displays [7]. Apart from their great potential for optoelectronic devices, $m$-plane InGaN/GaN QWs are a suitable platform to elucidate the complex mechanisms linked to carrier-density-dependent recombinations because of the absence of quantum confined Stark effect (QCSE) [8-14].

Since Auger recombination is often assumed to be the main reason for the efficiency droop, much effort has been devoted to the study of the Auger recombination process based on the so-called $A B C$ model [15]. Nevertheless, the main difficulty lies in identifying unambiguously the nonradiative coefficient $A$ and the bimolecular coefficient $B$, both being prerequisites to a proper determination of the Auger coefficient $C$. Due to the presence of unintentional $n$-type doping in InGaN/GaN QWs, both the experimentally deduced Shockley-Read-Hall

\footnotetext{
*Corresponding author: we.liu@epfl.ch

${ }^{\dagger}$ Corresponding author: gwenole.jacopin@epfl.ch
}

(SRH) recombination coefficient and the theoretical one reveal a saturation behavior upon increasing carrier density [16-19]. Contrary to what is often assumed, it is therefore inappropriate to use a constant $A$ coefficient. Likewise, the $B$ coefficient cannot be treated as a constant since, at high density, when the system gets degenerate, phase-space filling drastically reduces $B$ [20]. Moreover, considering the large binding energy of excitons in thin InGaN/GaN QWs (both polar and nonpolar ones), exciton recombination is expected to play an important role even at room temperature [21,22].

In this paper, using time-resolved photoluminescence (TRPL) with high temporal resolution (better than $100 \mathrm{ps}$ ), we study the density-dependent recombination dynamics in $m$-plane InGaN/GaN multiple QWs at different temperatures. We are able to determine both the effective lifetime through the decay of the signal and the radiative lifetime through the recording of the PL signal at initial time delays. By taking into account the $n$-type doping, phase-space filling, and the equilibrium between excitons and electron-hole pairs (e-h), we evaluate the density-dependent $A$ and $B$ coefficients, and obtain an upper limit for the $C$ coefficient in our sample. After a detailed description of the model accounting for densitydependent recombination dynamics, the experimental results are critically analyzed within the framework of this model.

\section{MODELING OF CARRIER-DENSITY-DEPENDENT RECOMBINATION DYNAMICS}

In order to model the carrier-density-dependent recombination dynamics, we start from the well-known $A B C$ model

$$
-\frac{d n}{d t}=A n+B n^{2}+C n^{3},
$$

where $n$ is the carrier density in the active region, the $A$ term takes into account SRH recombinations, $B$ is the bimolecular recombination coefficient which describes the radiative recombinations, and finally, the $C$ coefficient characterizes the dependence of the Auger recombinations as a function 
of carrier density. Though this model is widely used in the literature, it is only valid when the doping concentration $n_{0}$ and the injected carrier density $n$ are low enough $\left(n_{0}<10^{16} \mathrm{~cm}^{-3}\right.$ and $\left.n<10^{18} \mathrm{~cm}^{-3}\right)$. Indeed, at low injection $\left(n<10^{16} \mathrm{~cm}^{-3}\right)$, residual doping could play an important role [18]. Similarly, above $10^{18} \mathrm{~cm}^{-3}$, it has been shown that Fermi filling influences the carrier dynamics [23]. Since we investigate a sample with a relatively high $n$-type background density $\left(n_{0} \approx 10^{18} \mathrm{~cm}^{-3}\right.$, extracted from capacitance-voltage measurements) over a wide range of $n$ values (from $10^{15}$ to $10^{19} \mathrm{~cm}^{-3}$ ), the $A B C$ model should be modified accordingly. In addition, the $A B C$ model only describes the dynamics of e-h pairs recombination. In our case, the exciton binding energy $E_{b}$ is estimated to be larger than $26 \mathrm{meV}$ due to quantum confinement [24]. As a consequence, even at room temperature $\left(k_{B} T \approx 26 \mathrm{meV}\right)$, the influence of excitons needs to be taken into account. In the following, we will describe our modified $A B C$ model.

As discussed before, it has been proven that considering the $A$ coefficient as a constant is invalid as soon as the injected carrier density gets close to the residual doping one in InGaN/GaN QWs [19]. According to the SRH model, the lifetime of SRH recombinations $\tau_{\mathrm{nr}, \mathrm{SRH}}$ in presence of an $n$-type background doping should be written as $[16,17,19]$

$$
\tau_{\mathrm{nr}, \mathrm{SRH}}=\tau_{p 0}+\tau_{n 0} \frac{n_{\mathrm{e}-\mathrm{h}}}{n_{\mathrm{e}-\mathrm{h}}+n_{0}},
$$

where $\tau_{p 0}\left(\tau_{n 0}\right)$ is the hole (electron) capture time by deep level defects each one of them being occupied by one electron (hole), $n_{\mathrm{e}-\mathrm{h}}$ is the excess e-h plasma density (assuming $n_{\mathrm{e}-\mathrm{h}}=n$ for the moment), and $n_{0}$ is the $n$-type background density. The difference between $\tau_{p 0}$ and $\tau_{n 0}$ is mainly attributed to the different capture cross sections and velocities for electrons and holes.

Under high injection, the Auger recombination channel becomes the dominant nonradiative recombination mechanism and its lifetime $\tau_{\mathrm{nr} \text {, Auger }}$ is defined as

$\tau_{\mathrm{nr}, \text { Auger }}=\left[C_{\text {ehe }}\left(n_{0}+n_{\mathrm{e}-\mathrm{h}}\right)^{2}+C_{\mathrm{ehh}} n_{\mathrm{e}-\mathrm{h}}\left(n_{0}+n_{\mathrm{e}-\mathrm{h}}\right)\right]^{-1}$,

where $C_{\text {ehe }}\left(C_{\text {ehh }}\right)$ is the Auger recombination coefficient, which denotes that the energy from e-h recombinations is transferred to a third particle: electron (hole). It has been reported that the value of $C_{\text {ehe }}$ and that of $C_{\text {ehh }}$ are close to each other [25], we thus assume here for simplicity that $C_{\text {ehe }}=$ $C_{\text {ehh }}=C_{\text {Auger }}$. As a result, the total nonradiative lifetime of the e-h plasma, including SRH and Auger recombinations, is expressed as follows:

$$
\tau_{\mathrm{nr}, \mathrm{e}-\mathrm{h}}=\left(\tau_{\mathrm{nr}, \mathrm{SRH}}^{-1}+\tau_{\mathrm{nr}, \text { Auger }}^{-1}\right)^{-1} .
$$

Figure 1 shows the impact of the $n$-type background density on the lifetimes of SRH and Auger recombinations at $300 \mathrm{~K}$, with the parameters $\tau_{p 0}=1.4 \times 10^{-9} \mathrm{~s}, \tau_{n 0}=6 \times 10^{-9} \mathrm{~s}$, $C_{\text {Auger }}=1 \times 10^{-31} \mathrm{~cm}^{6} \mathrm{~s}^{-1}$ extracted from our experiment (see Sec. V). The upper $x$ axis denotes the two-dimensional (2D) $n_{\mathrm{e}-\mathrm{h}}$, considering the $3.3 \mathrm{~nm} \mathrm{QW}$ thickness of our sample. As shown in Fig. 1, upon increasing $n_{\mathrm{e}-\mathrm{h}}, \tau_{\mathrm{nr}, \mathrm{SRH}}$ shows two plateaus at $\tau_{p 0}$ for low densities and $\tau_{p 0}+\tau_{n 0}$ for high densities. The origin of such a behavior lies in: (1) the asymmetric electron and hole capture times and (2) the $n_{\mathrm{e}-\mathrm{h}}$ dependence of the fraction of defect levels occupied by electrons [17]. At low injection, most defect levels are

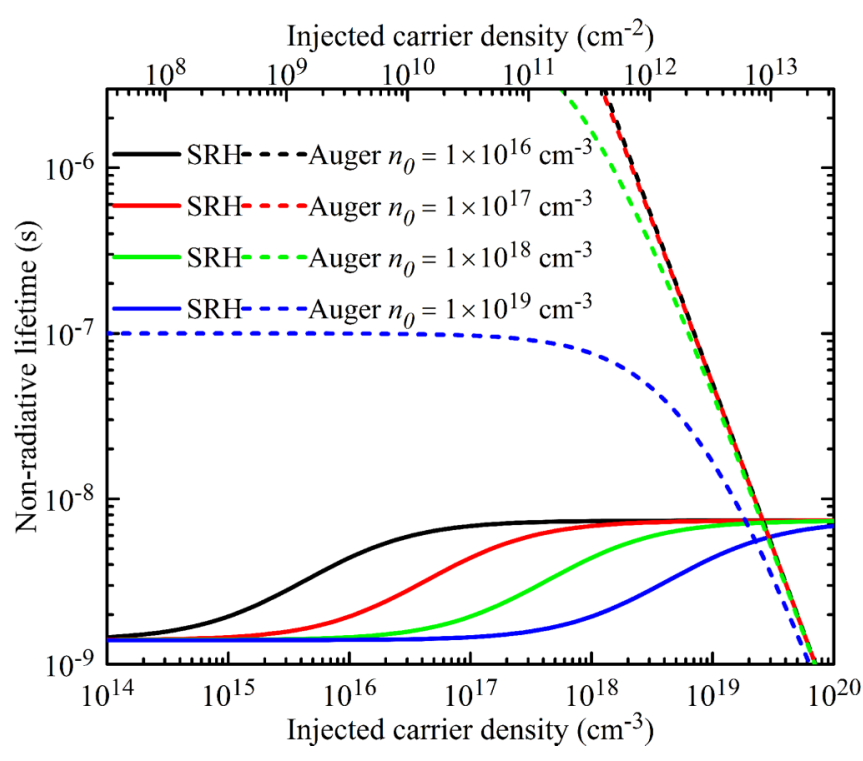

FIG. 1. Impact of different $n$-type background doping concentrations $\left(n_{0}\right)$ on the lifetimes of SRH (solid curves) and Auger (dashed curves) recombinations at $300 \mathrm{~K}$, obtained with the following parameters: hole capture time of defects $\tau_{p 0}=1.4 \times 10^{-9} \mathrm{~s}$, electron capture time of defects $\tau_{n 0}=6 \times 10^{-9} \mathrm{~s}$, and Auger recombination coefficient $C_{\text {Auger }}=1 \times 10^{-31} \mathrm{~cm}^{6} \mathrm{~s}^{-1}$.

occupied by background electrons, the annihilation of these electrons on the defect levels only depends on the hole capture time $\tau_{p 0}$. At high injection, the fraction of defect levels occupied by electrons decreases, because the photocarrier density exceeds the background doping. Consequently, for these deep levels, $\tau_{\mathrm{nr}, \mathrm{SRH}}$ becomes dependent on both the electron and hole capture time $\tau_{p 0}+\tau_{n 0}$, which can be one order of magnitude larger than $\tau_{p 0}$ in GaN-based QWs [19]. In addition, Fig. 1 demonstrates that Auger recombination only plays a role at very high injection $n>1 \times 10^{19} \mathrm{~cm}^{-3}$ and would be slightly enhanced by the $n$-type background doping when $n_{0}>1 \times 10^{19} \mathrm{~cm}^{-3}$.

Concerning the radiative lifetime of the e-h plasma $\tau_{r, \mathrm{e}-\mathrm{h}}$, instead of using the standard equation:

$$
1 /\left[B\left(n_{\mathrm{e}-\mathrm{h}}+n_{0}\right)\right]
$$

one should consider the phase-space filling effect, which leads to a drastic decrease in the $B$ term when the plasma becomes degenerate [23]. As a consequence, it is necessary to derive $\tau_{r, \mathrm{e}-\mathrm{h}}$ from Fermi's golden rule. The density-dependent $\tau_{r, \mathrm{e}-\mathrm{h}}$ is therefore described as follows:

$$
\begin{gathered}
R_{\mathrm{sp}}=\frac{n_{\mathrm{e}-\mathrm{h}}}{\tau_{r, \mathrm{e}-\mathrm{h}}} \\
R_{\mathrm{sp}}=\frac{1}{\tau_{0}} \int_{E_{\mathrm{QW}}}^{\infty} g_{2 \mathrm{D}}(E) f_{\mathrm{e}}(E) f_{\mathrm{h}}(E) d E \\
g_{2 \mathrm{D}}=\frac{\mu}{\pi \hbar^{2}} H\left(E-E_{\mathrm{QW}}\right), \\
f_{\mathrm{e}}=\left[\exp \left(\frac{\left(E-E_{\mathrm{QW}}\right) \mu / m_{\mathrm{e}}-E_{f, \mathrm{e}}}{k_{B} T}\right)+1\right]^{-1}, \\
f_{\mathrm{h}}=\left[\exp \left(\frac{\left(E-E_{\mathrm{QW}}\right) \mu / m_{\mathrm{h}}-E_{f, \mathrm{~h}}}{k_{B} T}\right)+1\right]^{-1},
\end{gathered}
$$



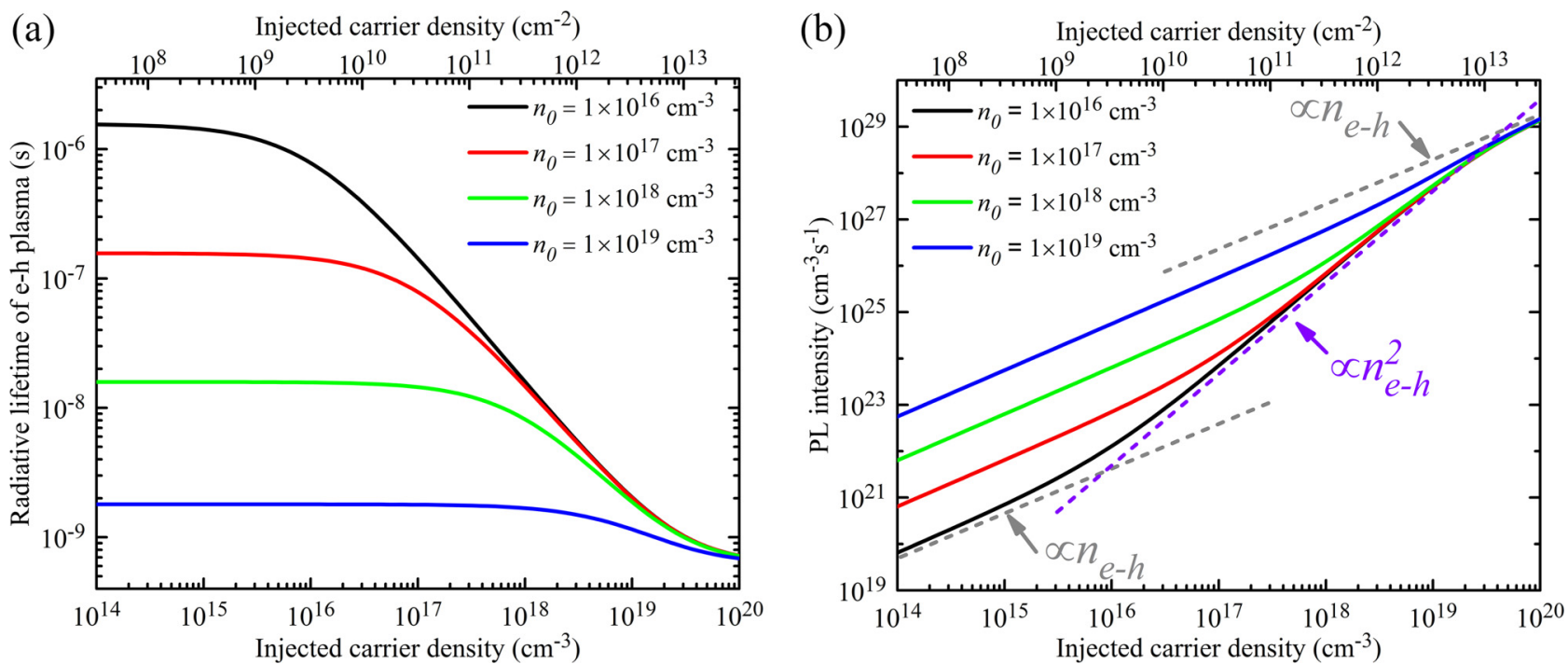

FIG. 2. (a) Injected carrier-density-dependent $\tau_{r, \mathrm{e}-\mathrm{h}}$ for different $n$-type background concentrations $\left(n_{0}\right)$ at $300 \mathrm{~K}$, with the average recombination time $\tau_{0}=6.2 \times 10^{-6} \mathrm{~s}$. (b) Corresponding injected carrier-density-dependent PL intensity given by $I_{\mathrm{pl}} \propto n_{\mathrm{e}-\mathrm{h}} / \tau_{r, \mathrm{e}-\mathrm{h}}$.

$$
\begin{gathered}
E_{f, \mathrm{e}}=k_{B} T \ln \left[\exp \left(\frac{\pi \hbar^{2}\left(n_{0}+n_{\mathrm{e}-\mathrm{h}}\right)}{m_{\mathrm{e}} k_{B} T}\right)-1\right], \\
E_{f, \mathrm{~h}}=k_{B} T \ln \left[\exp \left(\frac{\pi \hbar^{2} n_{\mathrm{e}-\mathrm{h}}}{m_{\mathrm{h}} k_{B} T}\right)-1\right], \\
\mu=m_{\mathrm{e}} m_{\mathrm{h}} /\left(m_{\mathrm{e}}+m_{\mathrm{h}}\right),
\end{gathered}
$$

where $R_{\mathrm{sp}}$ is the spontaneous recombination rate, $\tau_{r, \mathrm{e}-\mathrm{h}}$ is the radiative lifetime of the e-h plasma, $1 / \tau_{0}$ is the average recombination rate which depends on the oscillator strength, $g_{2 \mathrm{D}}$ is the two-dimensional (2D) joint density of states, $H\left(E-E_{\mathrm{QW}}\right)$ is the Heaviside step function, $E_{\mathrm{QW}}$ is the optical band gap of the QWs, $E$ is the emission energy, $f_{\mathrm{e}}\left(f_{\mathrm{h}}\right)$ is the Fermi-Dirac distribution for electrons (holes), $E_{f, \mathrm{e}}\left(E_{f, \mathrm{~h}}\right)$ is the quasi-Fermi level energy of electrons (holes), and $\mu$ is the in-plane carrier reduced mass. $m_{\mathrm{e}}=0.2 m_{0}$ is the effective electron mass and $m_{\mathrm{h}}=\sqrt{m_{\|} m_{\perp}}$ is the geometric mean value of the in-plane effective hole mass in the first valence band. By using k•p simulations [26], we estimate the effective mass along the $c$ axis to be $m_{\|}=1.83 m_{0}$ and the one in the perpendicular direction to be $m_{\perp}=0.185 m_{0}$. Note that we only consider the transition between the conduction band and the first valence band. It is a valid approximation because the energy splitting between the first and second valence bands in $m$-plane InGaN/GaN QWs (35 meV in the present sample as deduced from k·p simulations) [26] is larger than the thermal activation energy at $300 \mathrm{~K}$. In addition, $\hbar$ is the reduced Planck constant, and $m_{0}$ is the electron rest mass.

Figure 2(a) evidences the influence of the $n$-type background doping on the radiative lifetime of the e-h plasma at $300 \mathrm{~K}$. We see that $\tau_{r, \mathrm{e}-\mathrm{h}}$ can be significantly reduced when increasing $n_{0}$ at low injection. In addition, because of the effect of phase-space filling, $\tau_{r, \mathrm{e}-\mathrm{h}}$ saturates at high $n$ values around $1 \times 10^{20} \mathrm{~cm}^{-3}$. Figure 2(b) presents the corresponding PL intensity as a function of $n_{\mathrm{e}-\mathrm{h}}$ at $300 \mathrm{~K}$, defined as $I_{\mathrm{pl}} \propto R_{\mathrm{sp}}$. It clearly shows that the quadratic increase in $I_{\mathrm{pl}}$ as a function of $n_{\mathrm{e}-\mathrm{h}}$, following the bimolecular recombination mechanism, is only valid when $n_{\mathrm{e}-\mathrm{h}}$ lies in between $n_{0}$ and the degenerate density. These results justify the use of Fermi's golden rule to properly describe the e-h plasma radiative recombinations over a wide range of carrier densities.

As stated previously, considering the large exciton binding energy in $m$-plane InGaN/GaN QWs, it is necessary to evaluate the impact of excitons on the recombination dynamics at $300 \mathrm{~K}$. In our TRPL experiment, the equilibrium between excitons and the e-h plasma can be established by interparticle collisions within a few femtosecond time scale, which is much faster than the time scale for carrier recombination [27]. We therefore assume that equilibrium is established initially just after the excitation pulse. The ratio between exciton and the e-h plasma densities in a QW is usually determined by the standard Saha's equation derived from Boltzmann distribution [28]. This equation does not consider any Coulomb screening. Here we use instead the first order corrected Saha's equation (in the 2D case), which accounts for the Mott transition [29]:

$$
\begin{aligned}
\frac{\left(n_{\mathrm{e}-\mathrm{h}}+n_{0}\right) n_{\mathrm{e}-\mathrm{h}}}{n_{x}} & =\frac{\mu k_{B} T}{2 \pi \hbar^{2}} \frac{1}{\exp \left(E_{b} / k_{B} T\right)-1}, \\
n & =n_{x}+n_{\mathrm{e}-\mathrm{h}},
\end{aligned}
$$

where $n_{x}$ is the $2 \mathrm{D}$ density of excitons. Note that, due to Coulomb screening and phase-space filling effects, $E_{b}$ continuously decreases as $n$ increases [20]. To describe such a behavior in a simple way, we use an equation analogous to the Debye screening model [30]:

$$
E_{b}(n)=E_{b 0} \exp \left(-\frac{n}{n_{\text {Mott }}}\right),
$$

where $E_{b 0}$ is the exciton binding energy at low carrier density [24] and $n_{\text {Mott }}$ is the 2D critical Mott density, which is about $10^{12} \mathrm{~cm}^{-2}$ for GaN-based QWs [20,31]. Since we investigate InGaN QWs with a relatively low In content $(<10 \%)$, we assume that the critical Mott density remains unchanged.

In Fig. 3(a) we model the phase diagrams of the excitonic fraction at different injected carrier densities and 

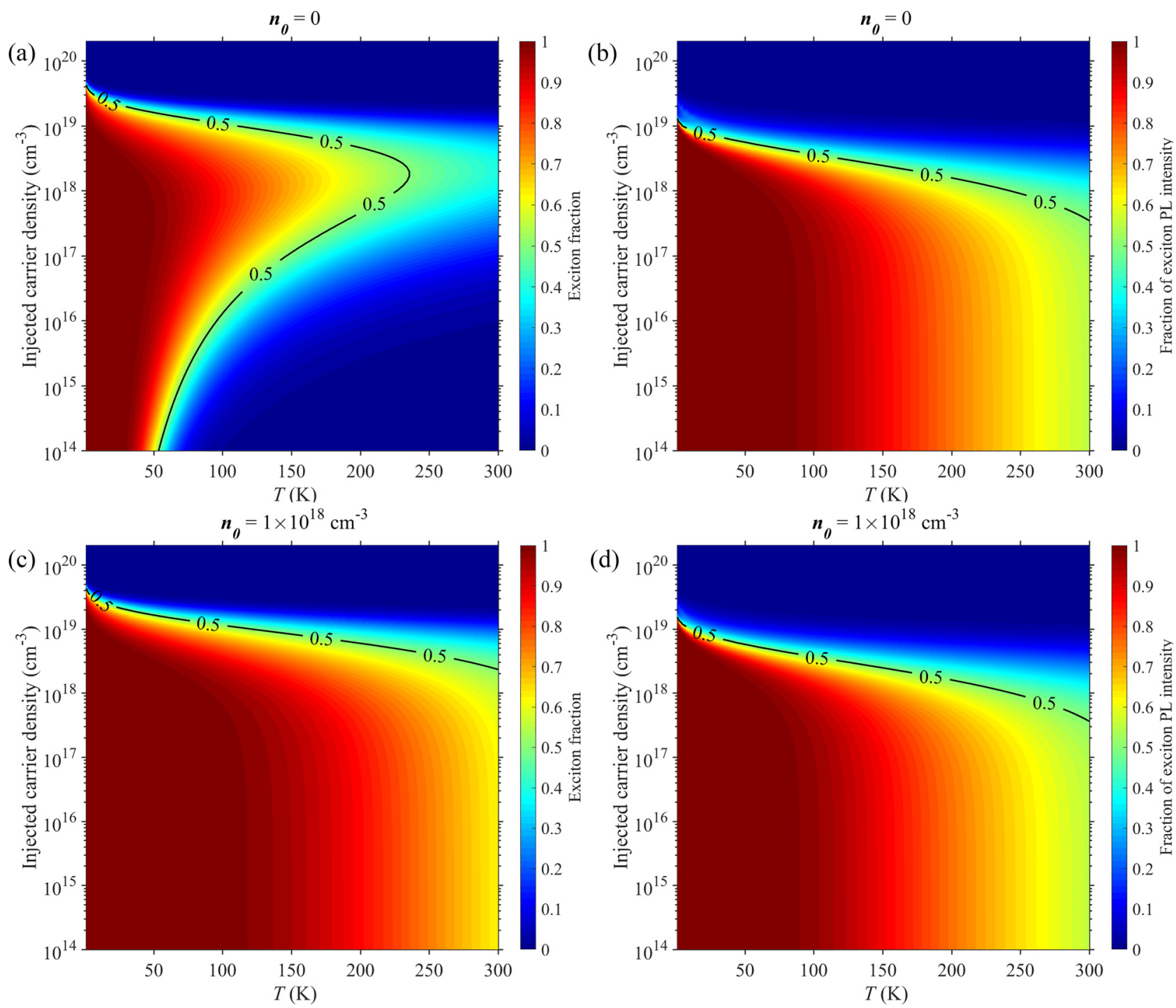

FIG. 3. (a) Computed phase diagrams of the exciton fraction vs total photoexcited carrier density in the QWs without $n$-type background doping, with the parameters: $n_{\text {Mott }}=1 \times 10^{12} \mathrm{~cm}^{-2}, E_{b 0}=42 \mathrm{meV}$. (b) Computed phase diagrams of the fraction of exciton PL intensity vs total PL intensity without $n$-type background doping, with the parameters: $n_{\text {Mott }}=1 \times 10^{12} \mathrm{~cm}^{-2}, E_{b 0}=42 \mathrm{meV}$. For simplicity, $\tau_{r, x}$ has been chosen to vary linearly between $1.7 \times 10^{-8} \mathrm{~s}$ at $300 \mathrm{~K}$ and $5.0 \times 10^{-10} \mathrm{~s}$ at $4 \mathrm{~K}$, and $\tau_{r, \mathrm{e}-\mathrm{h}}$ linearly decreases from $5 \times 10^{-6} \mathrm{~s}$ at 300 to $4 \mathrm{~K}$. Those values are extracted from our experiments (see Sec. V). (c) and (d) Corresponding phase diagrams with an $n$-type background doping $n_{0}=1 \times 10^{18} \mathrm{~cm}^{-3}$.

temperatures in the absence of any residual doping using $n_{\text {Mott }}=1 \times 10^{12} \mathrm{~cm}^{-2}$. We further determine $E_{b 0}=42 \mathrm{meV}$ through a self-consistent Schrödinger-Poisson modeling as described in Ref. [32]. This value is in agreement with that given in Ref. [33]. All the above-mentioned 2D densities are then transformed into three-dimensional ones, considering a quantum well thickness of $3.3 \mathrm{~nm}$. Figure 3(a) reveals that, when $n<n_{\text {Mott }}=3 \times 10^{18} \mathrm{~cm}^{-3}$ and $T<50 \mathrm{~K}$, the injected carrier population is mainly composed of excitons. At $300 \mathrm{~K}$, however, the maximum exciton fraction occurs for a total carrier density around $10^{18} \mathrm{~cm}^{-3}$ and then quickly vanishes. Figure 3(c) presents the phase diagram of the exciton fraction under an $n$-type background doping $n_{0}=1 \times 10^{18} \mathrm{~cm}^{-3}$, keeping all other parameters identical to those of Fig. 3(a). This reveals that the $n$-type background doping facilitates the formation of excitons, which leads to an exciton fraction up to
$63 \pm 6 \%$ at $300 \mathrm{~K}$. This naturally stems from the principle of Saha's equation: the equilibrium corresponds to the minimum of total free energy for the e-h plasma and the exciton gas.

Since the ratio between excitons and e-h plasma populations is determined by the first order corrected Saha's equation, we can further model the phase diagram of the fraction of exciton PL intensity by the following set of equations:

$$
\begin{gathered}
R_{x}(n, T)=\frac{n_{x} / \tau_{r, x}(T)}{n / \tau_{r}(T)}, \\
\tau_{r}(T)=\left(\frac{n_{x}}{n} \tau_{r, x}^{-1}(T)+\frac{n_{\mathrm{e}-\mathrm{h}}}{n} \tau_{r, \mathrm{e}-\mathrm{h}}^{-1}(T)\right)^{-1},
\end{gathered}
$$

where $R_{x}(n, T)$ is the fraction of the exciton PL intensity for a given set of $n$ and $T$ values. $\tau_{r}(T), \tau_{r, x}(T)$, and $\tau_{r, \mathrm{e}-\mathrm{h}}(T)$ are the effective radiative lifetime, the exciton radiative lifetime, 
and the e-h plasma radiative lifetime at $T$. Figures 3(b) and $3(d)$ present the phase diagrams of the fraction of exciton PL intensity without any $n$-type background doping and with $n_{0}=1 \times 10^{18} \mathrm{~cm}^{-3}$. First of all, it reveals that the $n$-type background doping does not change the phase diagrams of $R_{x}(n, T)$. Second, below the Mott density, the fraction of exciton PL intensity is independent of the injected density and only depends on temperature. To elucidate in a simple way the physics at stake, considering the low injection regime ( $\left.n \ll n_{\text {Mott }}\right), R_{x}(n, T)$ can be derived through Saha's Eqs. (14) and (5), as detailed below:

$$
R_{x}(T)=\frac{1 / \tau_{r, x}(T)}{B \cdot r(T)+1 / \tau_{r, x}(T)}
$$

and

$$
r(T)=\frac{\mu k_{B} T}{2 \pi \hbar^{2}} \frac{1}{\exp \left(E_{b 0} / k_{B} T\right)-1} .
$$

Thus, it is clear that $R_{x}(T)$ is independent of $n_{0}$ and $n$.

In the end, to properly model our experiments, and according to the usual definition of PL intensity decay:

$$
I_{\mathrm{pl}}(t) \propto \frac{n}{\tau_{r}} \exp \left(-\frac{t}{\tau_{\mathrm{eff}}}\right),
$$

the initial PL intensity $I_{\mathrm{pl}}(t=0)$ can be described as

$$
I_{\mathrm{pl}}(t=0) \propto \frac{n}{\tau_{r}},
$$

where a proportionality constant links experiment and modeling, which is only given by the TRPL system and does not change with either temperature or sample design. The effective lifetime $\tau_{\text {eff }}$ is expressed as

$$
\tau_{\text {eff }}=\left(\tau_{\mathrm{nr}}^{-1}+\tau_{r}^{-1}\right)^{-1} .
$$

The nonradiative lifetime $\tau_{\mathrm{nr}}$ and the radiative lifetime $\tau_{r}$ contain both the contributions from excitons and the e-h plasma, which are detailed below:

$$
\begin{aligned}
\tau_{\mathrm{nr}} & =\left(\frac{n_{x}}{n} \tau_{\mathrm{nr}, x}^{-1}+\frac{n_{\mathrm{e}-\mathrm{h}}}{n} \tau_{\mathrm{nr}, \mathrm{e}-\mathrm{h}}^{-1}\right)^{-1}, \\
\tau_{r} & =\left(\frac{n_{x}}{n} \tau_{r, x}^{-1}+\frac{n_{\mathrm{e}-\mathrm{h}}}{n} \tau_{r, \mathrm{e}-\mathrm{h}}^{-1}\right)^{-1},
\end{aligned}
$$

where $\tau_{r, x}$ and $\tau_{\mathrm{nr}, x}$ are the radiative and nonradiative lifetimes of excitons.

As a consequence, apart from the injected carrier density, the initial PL intensity only depends on the radiative lifetime, whereas the effective lifetime provides the relative contribution from the radiative and nonradiative recombinations. As our experiments are performed under identical conditions, a direct comparison of the PL intensities is meaningful.

\section{SAMPLE AND EXPERIMENTAL SETUPS}

The sample is grown by metal organic vapor phase epitaxy (MOVPE): the active layer consists of $5 \mathrm{InGaN} / \mathrm{GaN}$ (3.3 nm/12 nm) QWs with 8\%-9\% indium composition, capped by a 50 -nm-thick top $\mathrm{GaN}$ barrier. The $m$-plane free-standing $\mathrm{GaN}$ substrate is overgrown by a $1-\mu \mathrm{m}$-thick
GaN buffer layer prior to the growth of the QW region. In particular, to optimize the homogeneous distribution of indium in the QWs, the growth conditions of the QWs are set to $T=750^{\circ} \mathrm{C}$ and the V/III ratio $=10000$.

For PL experiments, the employed excitation source is a frequency-tripled Ti:sapphire laser (wavelength $\lambda=280 \mathrm{~nm}$, pulse width $\tau=2 \mathrm{ps}$, frequency $f=80.5 \mathrm{MHz}$ ). A pulse picker is employed to reduce $f$ by 22 times to avoid any heating of the sample. The excitation beam is focused on the sample by means of a UV objective having a focal length $F=13 \mathrm{~mm}$ and a numerical aperture NA $=0.32$. The PL spot is magnified 23 times by a second lens and focused on a pinhole of $150 \mu \mathrm{m}$ diameter, in order to only select the center of the excitation spot for having a uniform excitation power density. The PL signal is then recorded by a streak camera, which provides a temporal resolution better than 100 ps.

To estimate the power density impinging on our sample, supposing the laser beam has a Gaussian distribution of fluence, we first determine the full width at half maximum (FWHM $=0.8 \mathrm{~mm}$ ) as the diameter of the beam before the UV objective using the knife-edge method [34]. The radius of the focused laser beam on the sample is then estimated to be $5 \pm 1 \mu \mathrm{m}$. The beam transmission through the whole excitation path is about $52 \%$. The reflectance of the $\mathrm{GaN}$ surface, taking into account the refractive index of 2.6 at $280 \mathrm{~nm}$ [35], is estimated to be around 20\%. Since the absorption in the sample mainly happens in the first $\mathrm{GaN}$ capping layer and active layers, in our high quality sample (as will be evidenced in the following section), it is reasonable to assume that about half the carriers generated in the first capping layer are trapped by the QWs (the other half is assumed to diffuse to the surface), and all the carriers created in the active region are captured by the QWs. Therefore, the final fraction of carriers captured by the QWs is estimated to about $\sim 60 \%$, considering an $\mathrm{GaN}$ absorption coefficient equal to $1.5 \times 10^{5} \mathrm{~cm}^{-1}$ at $280 \mathrm{~nm}$ [36]. As a result, a $1 \mathrm{~mW}$ laser power at the output of the tripler finally generates a carrier density of about $6 \times 10^{19} \mathrm{~cm}^{-3}$ in the five QWs with $\sim 40 \%$ relative uncertainty, which arises from the accuracy of the estimation of the laser spot size and the absorption by the QWs and the barriers.

\section{EXPERIMENTAL RESULTS}

The first set of experiments relies on high power nonresonant TRPL performed at low temperature. In order to characterize the phase-space filling occurring under high excitation conditions, we probe the PL spectrum at early delay at $4 \mathrm{~K}$.

Figure 4(a) presents the power-dependent PL spectra measured at initial delay (between 0 and $20 \mathrm{ps).} \mathrm{The} \mathrm{laser}$ fluence on the sample ranges from 0.0075 to $22 \mu \mathrm{J} \mathrm{cm}^{-2}$. The main outcome is that, above $0.95 \mu \mathrm{J} \mathrm{cm}^{-2}$, the spectra become significantly broader as a result of phase-space filling. As shown in Fig. 4(b), below $0.95 \mu \mathrm{J} \mathrm{cm}^{-2}$, the FWHM of the PL spectra and the peak energy remain nearly constant around $38 \mathrm{meV}$ and $3.15 \mathrm{eV}$, respectively, whereas, above $0.95 \mu \mathrm{J} \mathrm{cm}^{-2}$, the FWHM gets wider by $20 \mathrm{meV}$ and the peak energy shifts toward higher energy by $6 \mathrm{meV}$. The significant spectral broadening and the slight blueshift in 


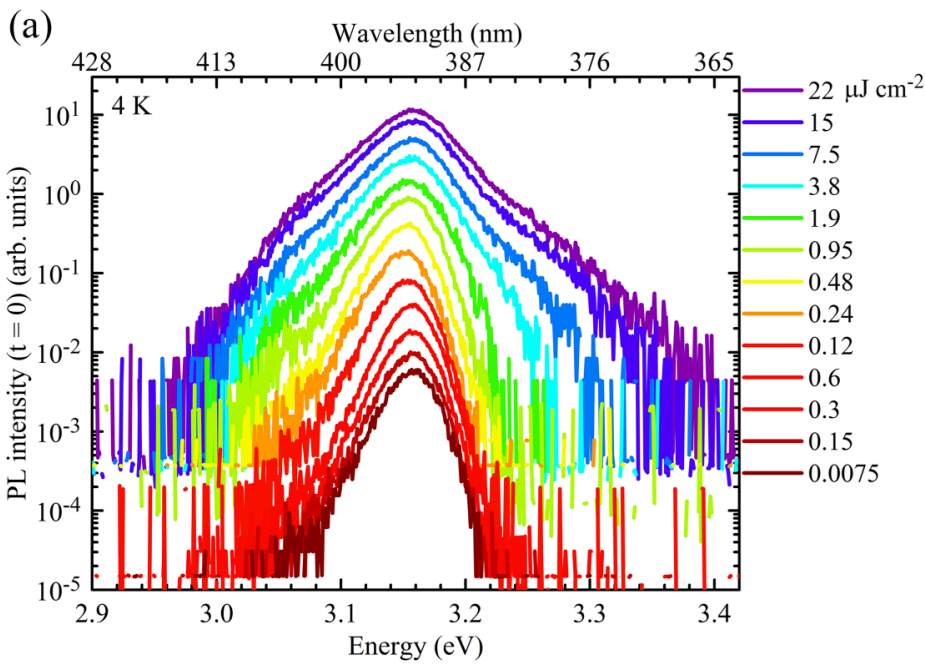

(b)

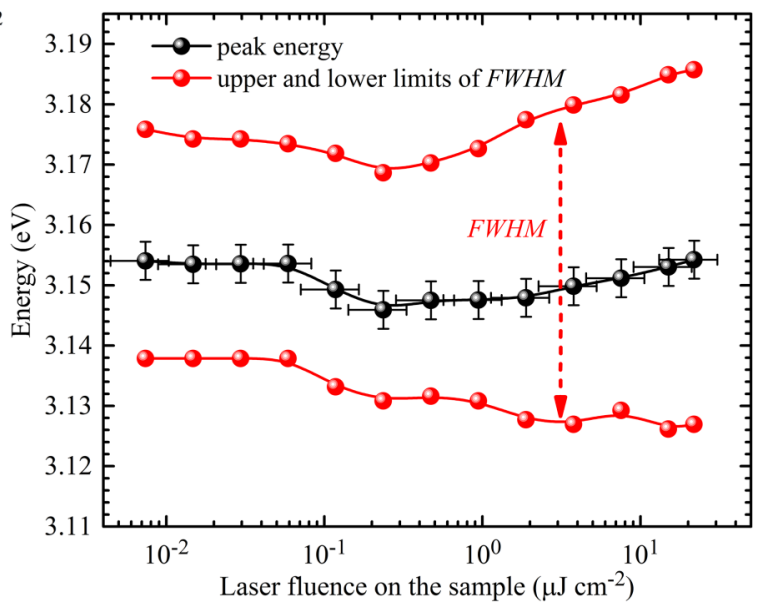

FIG. 4. (a) Power-dependent PL spectra at early time delay (between 0 and $20 \mathrm{ps)} \mathrm{at} 4 \mathrm{~K}$ for various laser fluences on the sample ranging from 0.0075 to $22 \mu \mathrm{J} \mathrm{cm}^{-2}$. (b) Corresponding power-dependent peak energies (black dots) and the upper and lower limits of the FWHM (red dots) of the PL spectra measured at initial delay.

the emission energy are the signatures of phase-space filling and provide further evidence of the fact that the initially injected carrier density is close to the Mott density, which is typically observed to occur between $5 \times 10^{11}$ and $2 \times$ $10^{12} \mathrm{~cm}^{-2}$ for GaN based QWs [20,31]. Since the temperature has a significant impact on the recombination dynamics and on the equilibrium between the exciton gas and the e-h plasma, we performed TRPL experiments at 4 and $300 \mathrm{~K}$ under identical conditions.

Figures 5(a) and 5(b) show the power-dependent decay curves integrated over the whole energy range of the QW emission spectra at 4 and $300 \mathrm{~K}$. Through the whole excitation range, $\tau_{\text {eff }}$ values are much longer at $300 \mathrm{~K}$ than they are at $4 \mathrm{~K}$. We can account for this observation on the basis of Eq. (23): the increase in the radiative lifetime $\tau_{r}$, caused by the spreading of the thermal distribution of the carriers, is more prominent than the decrease in the nonradiative lifetime $\tau_{\mathrm{nr}}[37]$.

(a)

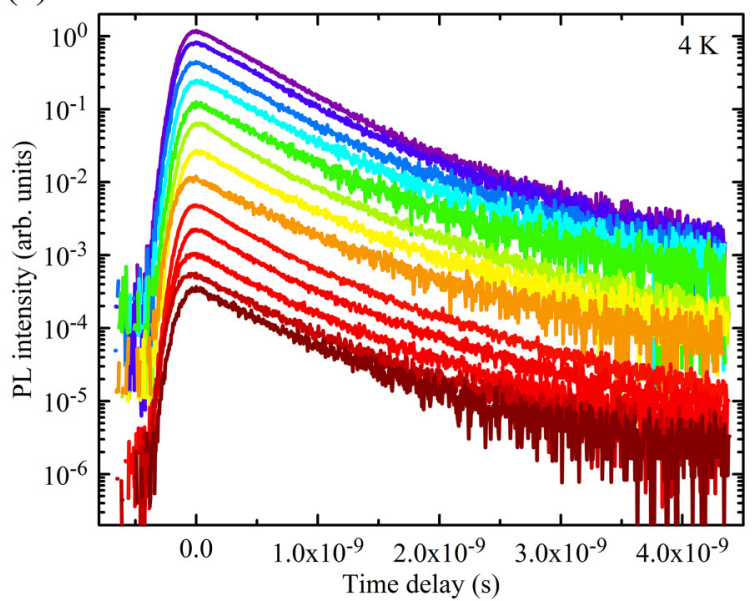

To get more insight into the recombination dynamics of carriers at early delays, we extract both the initial PL intensity $I_{\mathrm{pl}}(t=0)$ and the effective carrier lifetime $\tau_{\mathrm{eff}}$ by single exponential decay fitting at early delay (within $1 \mathrm{~ns}$ ). As mentioned before, $I_{\mathrm{pl}}(t=0)$ only depends on the radiative lifetime and the injected carrier density [37]. The high temporal resolution of our TRPL setup fulfils the requirements allowing us to probe the initial PL signal (see the Supplemental Material [38]).

In Fig. 6(a) we plot the injected carrier density $n$-dependent $I_{\mathrm{pl}}(t=0)$ at both $4 \mathrm{~K}$ (black dots) and $300 \mathrm{~K}$ (red dots). $I_{\mathrm{pl}}(t=0,4 \mathrm{~K})$ shows a linear dependence on $n$, which corresponds to a measured invariant effective lifetime $\tau_{\text {eff } 4 \mathrm{~K}} \approx$ $500 \mathrm{ps}$, as shown in Fig. 6(b). On the contrary, at $300 \mathrm{~K}$, the slope of $I_{\mathrm{pl}}(t=0,300 \mathrm{~K})$ changes from 1 to 2 above $n=10^{18} \mathrm{~cm}^{-3}$. Meanwhile, the effective lifetime $\tau_{\text {eff }, 300 \mathrm{~K}}$ first increases from 1.3 to $3.0 \mathrm{~ns}$ when $n<10^{18} \mathrm{~cm}^{-3}$, and then decreases down to $1.6 \mathrm{~ns}$ at $n=1 \times 10^{19} \mathrm{~cm}^{-3}$.

FIG. 5. (a) and (b) Power-dependent decay curves integrated over the whole energy range of QW emission spectra measured at 4 and $300 \mathrm{~K}$ under conditions otherwise identical for laser fluences on the sample ranging from 0.0075 to $22 \mu \mathrm{J} \mathrm{cm}^{-2}$. 


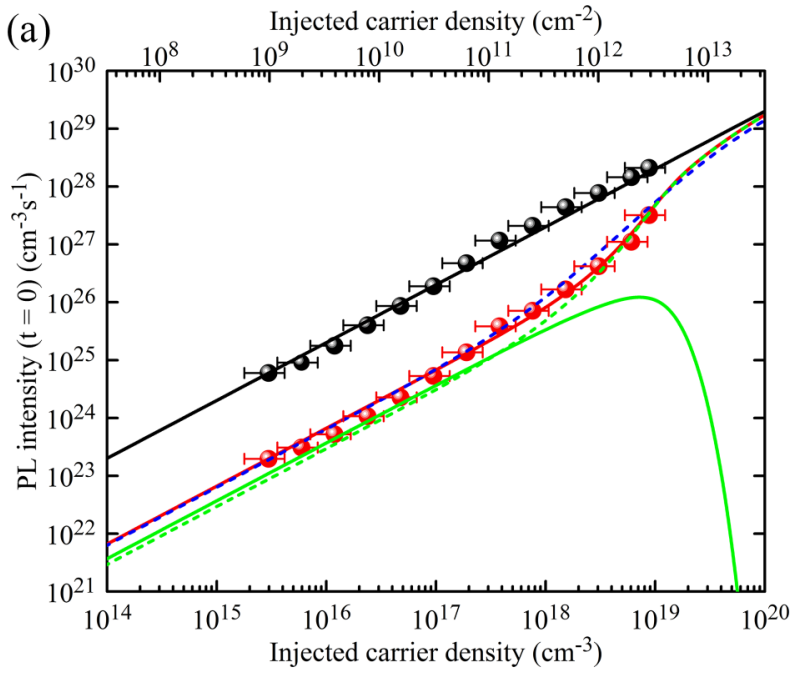

experimental $I_{p l}(t=0,4 \mathrm{~K})$
$-I_{p l}(t=0,4 \mathrm{~K})$ with Saha's eq. $\begin{aligned} & - \\ & -\end{aligned}$

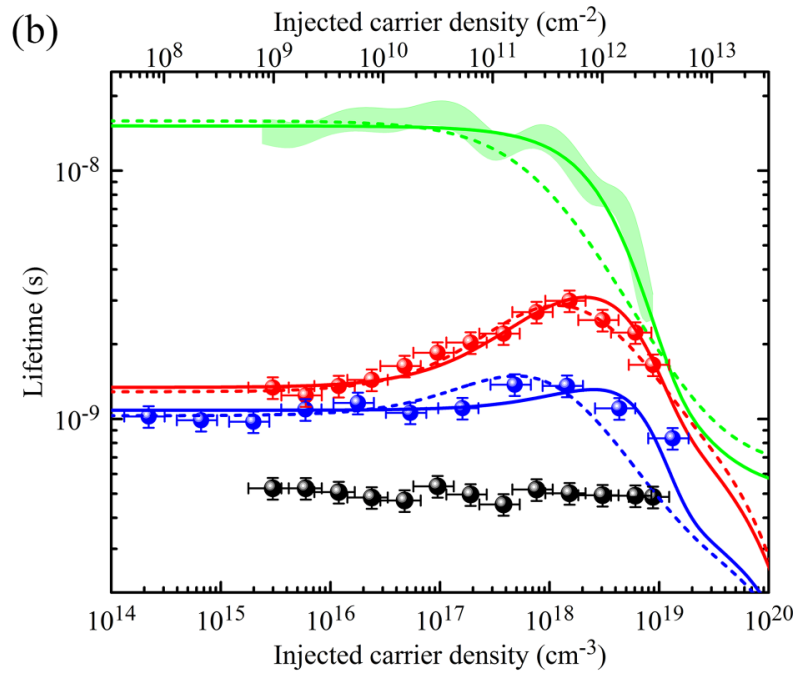

$$
\begin{aligned}
& \text { - experimental } \tau_{\text {eff,300 }} \mathrm{K} \quad \square \text { experimental } \tau_{r, 300 \mathrm{~K}} \\
& \text { - experimental } \tau_{\text {eff, } 200 \mathrm{~K}} \quad \text { experimental } \tau_{\text {eff, } 4 \mathrm{~K}}
\end{aligned}
$$

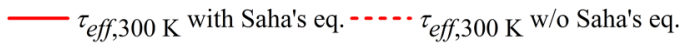

$$
\begin{aligned}
& \tau_{r, 300 \mathrm{~K}} \text { with Saha's eq. }-\cdots \tau_{r, 300 \mathrm{~K}} \text { w/o Saha's eq. }
\end{aligned}
$$

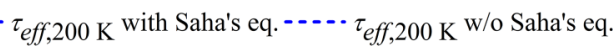

FIG. 6. (a) Injected carrier-density-dependent PL intensity at the initial delay $I_{\mathrm{pl}}(t=0)$ measured at $4 \mathrm{~K}$ (black dots) and $300 \mathrm{~K}(\mathrm{red}$ dots) extracted from the decay curves shown in Fig. 5 and related fits deduced from modeling with and without Saha's equation. The black solid line is the fit obtained by assuming that pure excitonic recombination is at play at $4 \mathrm{~K}$. Modeling with Saha's equation at $300 \mathrm{~K}$, the red solid curve is the total $I_{\mathrm{pl}}(t=0)$ containing the recombination of excitons and that of the e-h plasma, the green dashed curve is the e-h plasma $I_{\mathrm{pl}, \mathrm{e}-\mathrm{h}}(t=0)$, while the green solid curve is the exciton one $I_{\mathrm{pl}, x}(t=0)$. The blue dashed curve is the result of modeling without Saha's equation at $300 \mathrm{~K}$, namely, a pure e-h plasma system. (b) Corresponding injected carrier-density-dependent effective lifetimes $\tau_{\text {eff, } 4 \mathrm{~K}}$ (black dots) at $4 \mathrm{~K}, \tau_{\mathrm{eff}, 200 \mathrm{~K}}$ (blue dots) at $200 \mathrm{~K}, \tau_{\mathrm{eff}, 300 \mathrm{~K}}$ (red dots) at $300 \mathrm{~K}$, and the estimated radiative lifetime at $300 \mathrm{~K} \tau_{r, 300 \mathrm{~K}}$ (green shaded region). The solid curves are the related fits deduced from modeling using Saha's equation. The dashed curves are the related fits based on the modeling without Saha's equation. The error bar of the injected carrier density is $\sim 40 \%$. It is mainly coming from the estimation of the excitation spot diameter and the uncertainty on the injection efficiency $\eta_{\text {inj }}$ at room temperature. Concerning the precision on the effective lifetime, we estimate the error to be less than $10 \%$, due to the nonzero time windows ( $1 \mathrm{~ns})$ to estimate the effective lifetime at early delays.

To obtain a better understanding about these phenomena, we further extract the radiative lifetime using

$$
\frac{I_{\mathrm{pl}}(t=0,300 \mathrm{~K})}{I_{\mathrm{pl}}(t=0,4 \mathrm{~K})}=\eta_{\mathrm{inj}} \frac{\tau_{r, 4 \mathrm{~K}}}{\tau_{r, 300 \mathrm{~K}}},
$$

where $\tau_{r, 300 \mathrm{~K}}$ and $\tau_{r, 4 \mathrm{~K}}$ denote the radiative lifetime at a temperature of 300 and $4 \mathrm{~K}$, respectively, $\eta_{\mathrm{inj}}$ is the ratio of the injected carrier density at 300 and $4 \mathrm{~K}$, and $\tau_{r, 4 \mathrm{~K}}=$ $\tau_{\text {eff, } 4 \mathrm{~K}} \approx 500 \mathrm{ps}$ assuming that nonradiative recombinations are negligible at $4 \mathrm{~K}$. Since we use a nonresonant excitation scheme, the injection efficiency $\eta_{\text {inj }}$ may vary from 4 to $300 \mathrm{~K}$ because of the thermal activation of nonradiative defects in the barriers and recombinations occurring at the sample surface. According to Eq. (26), it may lead to a change in the estimation of the radiative lifetime at $300 \mathrm{~K}$. Here the upper limit of $\eta_{\text {inj }}$ is set to $100 \%$. The lower limit of $\eta_{\text {inj }}$ is obtained by fulfilling the lifetimes inequality $\tau_{r, 300 \mathrm{~K}} \geqslant \tau_{\text {eff, } 300 \mathrm{~K}}$. As a consequence, to satisfy the last inequality, the lower limit is bounded to $80 \%$. As shown in Fig. $6(\mathrm{~b}), \tau_{r, 300 \mathrm{~K}}$ is evolving within a region highlighted by the green shaded region, which stays approximately constant around $1.7 \times 10^{-8} \mathrm{~s}$ when $n<10^{18} \mathrm{~cm}^{-3}$, after which $\tau_{r, 300 \mathrm{~K}}$ reduces to about $2.5 \times 10^{-9} \mathrm{~s}$ at about $n=1 \times 10^{19} \mathrm{~cm}^{-3}$.
In order to estimate the contributions of residual doping, bimolecular recombination, and the presence of excitons, we model the recombination dynamics in the next section according to the formalism described in Sec. II.

\section{RESULTS OF THE MODELING AND DISCUSSION}

The experimental $I_{\mathrm{pl}}(t=0,4 \mathrm{~K})$ data can be fitted by only considering pure exciton recombination. To analyze the $300 \mathrm{~K}$ data, we start from $I_{\mathrm{pl}}(t=0,300 \mathrm{~K})$ and $\tau_{r, 300 \mathrm{~K}}$. The parameters that we have used are: $n_{0}=1 \times 10^{18} \mathrm{~cm}^{-3}$ as obtained from the capacitance-voltage measurements, $n_{\text {Mott }}=$ $1 \times 10^{12} \mathrm{~cm}^{-2}$ as measured in our previous reports [20,31], and $\tau_{r, x, 4 \mathrm{~K}}=\tau_{\mathrm{eff}, 4 \mathrm{~K}} \approx 500 \mathrm{ps}$. Finally, the unknown average recombination rate of the e-h plasma $1 / \tau_{0}$ is $2 \times 10^{5} \mathrm{~s}^{-1}$, as obtained from the best fit of the radiative lifetime under high injection. Similarly, the excitonic radiative lifetime is determined through the fitting of the experimental radiative lifetime under low injection $\tau_{r, x, 300 \mathrm{~K}}=1.7 \times$ $10^{-8} \mathrm{~s}$. The above-mentioned parameters are summarized in Table I.

As shown in Fig. 6(a), the fitting curve of $I_{\mathrm{pl}}(t=0,300 \mathrm{~K})$ (red solid curve) reproduces quite well the change in slope occurring above $10^{18} \mathrm{~cm}^{-3}$. We then decompose, 
TABLE I. Fitting parameters for the measured radiative recombination properties measured at $300 \mathrm{~K}$ based on Saha's equation.

\begin{tabular}{lccc}
\hline \hline$n_{0}\left(\mathrm{~cm}^{-3}\right)$ & $n_{\text {Mott }}\left(\mathrm{cm}^{-2}\right)$ & $\tau_{0}(\mathrm{~s})$ & $\tau_{x, r}(\mathrm{~s})$ \\
\hline $1 \times 10^{18}$ & $1 \times 10^{12}$ & $5 \times 10^{-6}$ & $1.7 \times 10^{-8}$ \\
\hline \hline
\end{tabular}

through Saha's equation, the total $I_{\mathrm{pl}}(t=0,300 \mathrm{~K})$ into e-h plasma PL intensity $I_{\mathrm{pl}, \mathrm{e}-\mathrm{h}}(t=0,300 \mathrm{~K})$ and exciton PL intensity $I_{\mathrm{pl}, x}(t=0,300 \mathrm{~K})$. This allows us to show that $I_{\mathrm{pl}}(t=0,300 \mathrm{~K})$ is dominated by $I_{\mathrm{pl}, \mathrm{e}-\mathrm{h}}(t=0,300 \mathrm{~K})$ [green dashed curve in Fig. 6(a)]. The change in slope of $I_{\mathrm{pl}, \mathrm{e}-\mathrm{h}}(t=0,300 \mathrm{~K})$ from 1 to 2 is mainly due to the injected carrier density exceeding the $n$-type background doping one. To be more specific, the recombination rate at low injection is given by $I_{\mathrm{pl}}(n)=B n_{0} n_{\mathrm{e}-\mathrm{h}}$ and the slope is equal to one. When the injected carrier density exceeds $n_{0}$, it transforms into $I_{\mathrm{pl}}(n)=B n_{\mathrm{e}-\mathrm{h}}^{2}$ and the slope becomes equal to 2. Fermi's golden rule further predicts that the slope of $I_{\mathrm{pl}, \mathrm{e}-\mathrm{h}}(t=0,300 \mathrm{~K})$ will return to 1 for $n$ above $1 \times 10^{20} \mathrm{~cm}^{-3}$, because of phase-space filling.

On the other hand, the exciton dynamics around the Mott transition region is featured by $I_{\mathrm{pl}, x}(t=0,300 \mathrm{~K}$ ) (green solid line) in Fig. 6(a). The maximum of $I_{\mathrm{pl}, x}(t=0,300 \mathrm{~K})$ occurs around $1 \times 10^{19} \mathrm{~cm}^{-3}$, which corresponds to the lower boundary of the Mott transition region. When $n>1 \times$ $10^{19} \mathrm{~cm}^{-3}$, the drastic collapse in $I_{\mathrm{pl}, x}(t=0,300 \mathrm{~K})$ is due to exciton dissociation occurring through Coulomb screening. In addition, Saha's equation suggests that, at low injection densities, $63 \pm 6 \%$ of the injected carrier population form excitons, yet the corresponding weight of $I_{\mathrm{pl}, x}(t=0,300 \mathrm{~K})$ is $56 \pm 10 \%$.

In Fig. 6(b), the good correspondence between the fit (green solid curve) and experimental data (green shaded region) of $\tau_{r, 300 \mathrm{~K}}$ further attests the validity of our model. In brief, the nearly constant $\tau_{r, 300 \mathrm{~K}} \approx 1.7 \times 10^{-8} \mathrm{~s}$ when $n<$ $1 \times 10^{18} \mathrm{~cm}^{-3}$ results from the rather large $n$-type background doping and the presence of an exciton population. Between $n=1 \times 10^{18}$ and $1 \times 10^{19} \mathrm{~cm}^{-3}$, the rapid decrease in $\tau_{r, 300 \mathrm{~K}}$ is due to the enhancement of bimolecular recombination.

The previous determination of $\tau_{r, 300 \mathrm{~K}}$ allows us to analyze further and unambiguously the effective nonradiative carrier lifetime $\tau_{\mathrm{nr}, 300 \mathrm{~K}}$. As mentioned before, the increasing $\tau_{\text {eff, } 300 \mathrm{~K}}$ at low injection is interpreted as the saturation of SRH recombinations. As for the exciton nonradiative lifetime $\tau_{\mathrm{nr}, x}$, we assume that it mainly depends on the trapping of the carrier with the longer capture time: in our case, $\tau_{\mathrm{nr}, x}=\tau_{n 0}$ [39]. As a result, we are able to determine the hole capture time, $\tau_{p 0}=6.5 \times 10^{-10} \mathrm{~s}$, via the fit of $\tau_{\text {eff }, 300 \mathrm{~K}}$ at low injection. The electron capture time $\tau_{n 0}=6 \times 10^{-9} \mathrm{~s}$ is further determined by fitting $\tau_{\text {eff, } 300 \mathrm{~K}}$ at higher injection. The only parameter left is the Auger coefficient $C_{\text {Auger }}$ which is used to match the decreasing trend of $\tau_{\text {eff, } 300 \mathrm{~K}}$ when the carrier density is above $n>1 \times 10^{18} \mathrm{~cm}^{-3}$. It turns out that $C_{\text {Auger }}$ should be less than $1 \times 10^{-31} \mathrm{~cm}^{6} \mathrm{~s}^{-1}$, since the decrease in $\tau_{\mathrm{eff}, 300 \mathrm{~K}}$ is mainly dominated by $\tau_{r, 300 \mathrm{~K}}$ in our sample. This result is consistent with theoretical works that state that decreasing the weight of disorder reduces Auger recombination [25]. The above fitting parameters, allowing us to reproduce the observed vari-
TABLE II. Fitting parameters related to nonradiative recombinations at $300 \mathrm{~K}$ based on Saha's equation.

\begin{tabular}{cccc}
\hline \hline$\tau_{\mathrm{nr}, x}(\mathrm{~s})$ & $\tau_{n 0}(\mathrm{~s})$ & $\tau_{p 0}(\mathrm{~s})$ & $C_{\text {Auger }}\left(\mathrm{cm}^{6} \mathrm{~s}^{-1}\right)$ \\
\hline $6 \times 10^{-9}$ & $6 \times 10^{-9}$ & $6.5 \times 10^{-10}$ & $1 \times 10^{-31}$ \\
\hline \hline
\end{tabular}

ations in $\tau_{\text {eff, } 300 \mathrm{~K}}$ related to nonradiative recombinations, are summarized in Table II. Finally, the curve given by the model for $\tau_{\text {eff, } 300 \mathrm{~K}}$ (red solid line) reproduces very satisfactorily the experiment, and demonstrates that the reduction in $\tau_{\text {eff, } 300 \mathrm{~K}}$ between $n=1 \times 10^{18}$ and $1 \times 10^{19} \mathrm{~cm}^{-3}$ is dominated by radiative recombinations.

As a comparison, to illustrate the impact of excitons on the recombination dynamics at $300 \mathrm{~K}$, we further model the change in lifetime assuming that all the injected carriers stay in the form of an e-h plasma, while keeping the same $n_{0}, n_{\text {Mott }}$, $\tau_{n 0}$, and $C_{\text {Auger }}$ values used in the model with Saha's equation at $300 \mathrm{~K}$, but with different $\tau_{0}=6.2 \times 10^{-6} \mathrm{~s}$ and $\tau_{p 0}=$ $1.4 \times 10^{-9} \mathrm{~s}$. As shown in Fig. 6(a), even though the pure e-h plasma contribution $I_{\mathrm{pl}}(t=0,300 \mathrm{~K})$ (blue dashed line) reproduces accurately the experimental data below the Mott transition, it is slightly above the experimental data in the Mott transition region. Correspondingly, in Fig. $6(\mathrm{~b}), \tau_{r, 300 \mathrm{~K}}$ (green dashed line) derived from a pure e-h plasma model is shorter than the experimental one. Considering the fitting procedure and the analog radiative lifetime $\tau_{r, \mathrm{e}-\mathrm{h}, 300 \mathrm{~K}}=1 /\left(B n_{0}\right)$ at low injection, the $B$ term (related to $\tau_{0}$ in Fermi's golden rule) is determined by both the known $n_{0}$ value and the nearly constant radiative lifetime at low injection. Therefore, in the Mott transition region, the discrepancy in the fitting of $\tau_{r, \mathrm{e}-\mathrm{h}, 300 \mathrm{~K}}$ results from an overestimated $n_{\mathrm{e}-\mathrm{h}}$ value if the formation of excitons is neglected, even though the reduction in $B$ by phase-space filling has been properly considered.

To observe more pronounced excitonic effects and confirm our conclusions, we further perform experiments at $200 \mathrm{~K}$ and fit the $\tau_{\text {eff, } 200 \mathrm{~K}}$ dependence using the two possible models as highlighted above. For the fit of a pure e-h plasma at $200 \mathrm{~K}$, $\tau_{0}=2.3 \times 10^{-6} \mathrm{~s}$, which is the only fitting parameter different from the one used at $300 \mathrm{~K}$. For the fit with Saha's equation at $200 \mathrm{~K}$, we take $\tau_{0}=2.6 \times 10^{-6} \mathrm{~s}$ and $\tau_{r, x}=1.6 \times 10^{-9} \mathrm{~s}$. As shown in Fig. 6(b), the pure e-h plasma scenario (blue dashed curve) cannot fit $\tau_{\text {eff, } 200 \mathrm{~K}}$ in the Mott density region, which strongly indicates the overestimation of $n_{\mathrm{e}-\mathrm{h}}$. Conversely, the model with Saha's equation reproduces very well the smooth reduction in $\tau_{\text {eff }, 200 \mathrm{~K}}$ in the Mott transition region. At $200 \mathrm{~K}$, $87 \pm 5 \%$ of the injected carrier density is bound into excitons when $n<1 \times 10^{18} \mathrm{~cm}^{-3}$, and then gradually reduces to $\sim 30 \%$ at $n=1 \times 10^{19} \mathrm{~cm}^{-3}$, due to Coulomb screening. In addition, the weight of $I_{\mathrm{pl}, x}(t=0,200 \mathrm{~K})$ is supposed to be $95 \pm 2 \%$, compared to $56 \pm 10 \%$ of that at $300 \mathrm{~K}$. Therefore, exciton recombination is far more prominent at $200 \mathrm{~K}$.

As shown in Fig. 7 (a), when using the above-mentioned model, we are able to derive the injected carrier-densitydependent internal quantum efficiency (red shaded region), defined as IQE $=\tau_{\text {eff }, 300 \mathrm{~K}} / \tau_{r, 300 \mathrm{~K}}$. The model with Saha's equation gives a better fit (red solid curve) compared to the one based on a pure e-h plasma (red dashed curve). Both models predict a similar decrease in IQE due to Auger recombination 

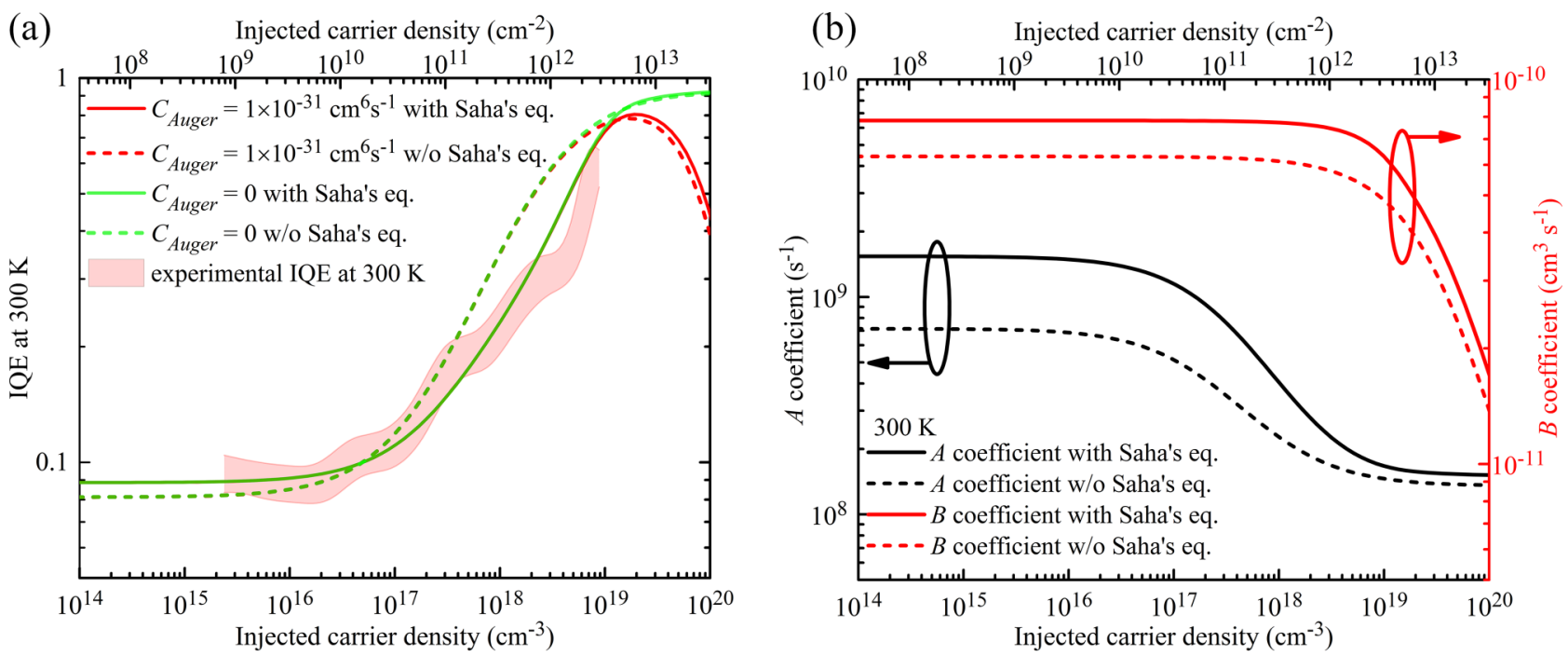

FIG. 7. (a) Injected carrier-density-dependent internal quantum efficiency derived from IQE $=\tau_{\text {eff, } 300 \mathrm{~K}} / \tau_{r, 300 \mathrm{~K}}$ (red shaded region). The red solid and red dashed curves are fits based on models with and without Saha's equation, the fitting parameters of which are the same as the fits shown in Fig. 6. The green solid curve and the green dashed curve are the fits based on models with and without Saha's equation, keeping the same fitting parameters as for the previous fits except for $C_{\text {Auger }}=0$. (b) Injected carrier-density-dependent $B$ coefficients (red curves) and $A$ coefficients (black curves) extracted from the experimental data shown in Fig. 6 . The solid (dashed) curves are fits based on the model with and without Saha's equation, respectively.

at high injection density, for $C_{\text {Auger }}=1 \times 10^{-31} \mathrm{~cm}^{6} \mathrm{~s}^{-1}$. As a comparison, setting $C_{\text {Auger }}=0$ for both models, with and without Saha's equation (green solid and dashed curves) predict IQEs larger than $90 \%$ at high injection. The densitydependent $A$ and $B$ coefficients can be further derived by $A=1 / \tau_{\mathrm{nr}, \text { SRH. } 300 \mathrm{~K}}$ and $B=1 /\left[\left(n_{0}+n_{\mathrm{e}-\mathrm{h}}\right) \tau_{r, \mathrm{e}-\mathrm{h}, 300 \mathrm{~K}}\right]$, as presented in Fig. 7(b). Note that getting $A$ and $B$ coefficients in a convincing way is a prerequisite to properly determine $C_{\text {Auger }}$. Therefore our experiments and the model including Saha's equation provide an effective way to analyze in better detail the Auger related efficiency droop at high injection in GaN-based heterostructures.

\section{CONCLUSIONS}

In conclusion, based on high injection time-resolved photoluminescence experiments, we have studied the carrierdensity-dependent recombination dynamics in $m$-plane InGaN/GaN QWs with a large $n$-type background doping $(\sim 1 \times$ $\left.10^{18} \mathrm{~cm}^{-3}\right)$. At low temperature, pure excitonic recombination is evidenced, even under high injection conditions, through a nearly invariant effective lifetime of about 500 ps. Thanks to the high temporal resolution of our setup, we are able to probe the recombination dynamics even at very early times after injection, which allows us to extract the carrier-densitydependent radiative lifetime at all temperatures. As a result, we could extract convincingly the injected carrier-density- dependent bimolecular recombination coefficient, taking into account the $n$-type background doping, phase-space filling effects, equilibrium between excitons and the e-h plasma, and the Mott transition. Saha's equation reveals that the $n$-type background doping not only facilitates bimolecular recombination at low injection, but also promotes the formation of excitons. The validity of Saha's equation is further attested by its ability to reproduce the carrier-density-dependent effective lifetime at $200 \mathrm{~K}$ where stronger excitonic effects take place. Based on the SRH model, we could extract the electron and hole capture times by deep levels at $300 \mathrm{~K}$. We have also demonstrated that the increasing effective lifetime with increasing carrier density at $300 \mathrm{~K}$ stems from asymmetric capture times between electrons and holes and the presence of an $n$-type background doping. The SRH model also predicts that, in QWs with a heavier $n$-type background doping, higher injection will be required to saturate the SRH recombination. Finally, because of the reasonable determination of densitydependent $A$ and $B$ coefficients in the Mott transition region, our method provides a way to evaluate the impact of Auger recombination.

\section{ACKNOWLEDGMENT}

The present work was financially supported by the Swiss National Science Foundation under Projects No. 153620 and No. 154853.
[1] S. P. Denbaars, D. Feezell, K. Kelchner, S. Pimputkar, C. C. Pan, C. C. Yen, S. Tanaka, Y. Zhao, N. Pfaff, R. Farrell, M. Iza, S. Keller, U. Mishra, J. S. Speck, and S. Nakamura, Acta Mater. 61, 945 (2013).
[2] M.-H. Chang, D. Das, P. V. Varde, and M. Pecht, Microelectron. Reliab. 52, 762 (2012).

[3] S. F. Chichibu, A. C. Abare, M. P. Mack, M. S. Minsky, T. Deguchi, D. Cohen, P. Kozodoy, S. B. Fleischer, S. Keller, 
J. S. Speck, J. E. Bowers, E. Hu, U. K. Mishra, L. A. Coldren, S. P. DenBaars, K. Wada, T. Sota, and S. Nakamura, Mater. Sci. Eng. B 59, 298 (1999).

[4] J. Cho, E. F. Schubert, and J. K. Kim, Laser Photon. Rev. 7, 408 (2013).

[5] P. Waltereit, O. Brandt, A. Trampert, H. T. Grahn, J. Menniger, M. Ramsteiner, M. Reiche, and K. H. Ploog, Nature 406, 865 (2000).

[6] K.-C. Kim, M. C. Schmidt, H. Sato, F. Wu, N. Fellows, Z. Jia, M. Saito, S. Nakamura, S. P. DenBaars, J. S. Speck, and K. Fujito, Appl. Phys. Lett. 91, 181120 (2007).

[7] E. Matioli, S. Brinkley, K. M. Kelchner, Y.-L. Hu, S. Nakamura, S. DenBaars, J. Speck, and C. Weisbuch, Light Sci. Appl. 1, e22 (2012).

[8] G. A. Garrett, H. Shen, M. Wraback, A. Tyagi, M. C. Schmidt, J. S. Speck, S. P. DenBaars, and S. Nakamaura, Phys. Status Solidi C 6, S800 (2009).

[9] Y. Huang, K. W. Sun, A. M. Fischer, Q. Y. Wei, R. Juday, F. A. Ponce, R. Kato, and T. Yokogawa, Appl. Phys. Lett. 98, 261914 (2011).

[10] S. Marcinkevičius, K. M. Kelchner, L. Y. Kuritzky, S. Nakamura, S. P. DenBaars, and J. S. Speck, Appl. Phys. Lett. 103, 111107 (2013).

[11] S. Marcinkevičius, K. M. Kelchner, S. Nakamura, S. P. DenBaars, and J. S. Speck, Phys. Status Solidi C 11, 690 (2014).

[12] D. Sutherland, T. Zhu, J. T. Griffiths, F. Tang, P. Dawson, D. Kundys, F. Oehler, M. J. Kappers, C. J. Humphreys, and R. A. Oliver, Phys. Status Solidi B 252, 965 (2015).

[13] T. Langer, M. Klisch, F. Alexej Ketzer, H. Jönen, H. Bremers, U. Rossow, T. Meisch, F. Scholz, and A. Hangleiter, Phys. Status Solidi B 253, 133 (2016).

[14] P. Dawson, S. Schulz, R. A. Oliver, M. J. Kappers, and C. J. Humphreys, J. Appl. Phys. 119, 181505 (2016).

[15] J. Iveland, L. Martinelli, J. Peretti, J. S. Speck, and C. Weisbuch, Phys. Rev. Lett. 110, 177406 (2013).

[16] R. N. Hall, Phys. Rev. 87, 387 (1952).

[17] W. Shockley and W. T. Read, Phys. Rev. 87, 835 (1952).

[18] O. Brandt, H. Yang, and K. H. Ploog, Phys. Rev. B 54, R5215 (1996).

[19] T. Langer, A. Chernikov, D. Kalincev, M. Gerhard, H. Bremers, U. Rossow, M. Koch, and A. Hangleiter, Appl. Phys. Lett. 103, 202106 (2013).

[20] G. Rossbach, J. Levrat, G. Jacopin, M. Shahmohammadi, J.-F. Carlin, J.-D. Ganière, R. Butté, B. Deveaud, and N. Grandjean, Phys. Rev. B 90, 201308(R) (2014).
[21] A. Hangleiter, Z. Jin, M. Gerhard, D. Kalincev, T. Langer, H. Bremers, U. Rossow, M. Koch, M. Bonn, and D. Turchinovich, Phys. Rev. B 92, 241305 (2015).

[22] A. Hangleiter, T. Langer, M. Gerhard, D. Kalincev, A. Kruse, H. Bremers, U. Rossow, and M. Koch, Proc. SPIE 9363, 93631R (2015).

[23] A. David and M. J. Grundmann, Appl. Phys. Lett. 96, 103504 (2010).

[24] S. Khatsevich and D. H. Rich, J. Phys. Condens. Matter 20, 215223 (2008).

[25] E. Kioupakis, D. Steiauf, P. Rinke, K. T. Delaney, and C. G. Van de Walle, Phys. Rev. B 92, 035207 (2015).

[26] L. Schade, T. Wernicke, J. Rass, S. Ploch, M. Weyers, M. Kneissl, and U. T. Schwarz, Phys. Status Solidi B 253, 145 (2016).

[27] R. Huber, F. Tauser, A. Brodschelm, M. Bichler, G. Abstreiter, and A. Leitenstorfer, Nature 414, 286 (2001).

[28] P. Corfdir, J. Levrat, G. Rossbach, R. Butté, E. Feltin, J.F. Carlin, G. Christmann, P. Lefebvre, J.-D. Ganière, N. Grandjean, and B. Deveaud-Plédran, Phys. Rev. B 85, 245308 (2012).

[29] D. Snoke, Solid State Commun. 146, 73 (2008).

[30] L.-X. Zhai, Y. Wang, and J.-J. Liu, J. Appl. Phys. 112, 033709 (2012).

[31] M. Shahmohammadi, G. Jacopin, G. Rossbach, J. Levrat, E. Feltin, J.-F. Carlin, J.-D. Ganière, R. Butté, N. Grandjean, and B. Deveaud, Nat. Commun. 5, 5251 (2014).

[32] G. Rossbach, J. Levrat, E. Feltin, J.-F. Carlin, R. Butté, and N. Grandjean, Phys. Rev. B 88, 165312 (2013).

[33] M. Funato and Y. Kawakami, J. Appl. Phys. 103, 093501 (2008).

[34] A. E. Siegman, M. W. Sasnett, and T. F. Johnston, IEEE J. Quantum Electron. 27, 1098 (1991).

[35] H. Amano, N. Watanabe, N. Koide, and I. Akasaki, Jpn. J. Appl. Phys. 32, L1000 (1993).

[36] J. F. Muth, J. H. Lee, I. K. Shmagin, R. M. Kolbas, H. C. Casey, B. P. Keller, U. K. Mishra, and S. P. DenBaars, Appl. Phys. Lett. 71, 2572 (1997).

[37] P. Corfdir, J. Levrat, A. Dussaigne, P. Lefebvre, H. Teisseyre, I. Grzegory, T. Suski, J.-D. Ganière, N. Grandjean, and B. Deveaud-Plédran, Phys. Rev. B 83, 245326 (2011).

[38] See Supplemental Material at http://link.aps.org/supplemental/ 10.1103/PhysRevB.94.195411 which provides the fluence dependent TRPL at $6 \mathrm{~K}$ recorded by synchroscan mode.

[39] A. Alkauskas, C. E. Dreyer, J. L. Lyons, and C. G. Van de Walle, Phys. Rev. B 93, 201304 (2016). 